\title{
A Low Complexity Time Domain Spectrum Sensing Technique for OFDM
}

\author{
(Invited Paper) \\ Xianbin Wang, Hao Li, Serguei Primak and Viet-Ha Pham \\ Department of Electrical and Computer Engineering, \\ The University of Western Ontario, London, Ontario, Canada
}

\begin{abstract}
Due to the widespread deployment of OFDM and recent advent of cognitive radio communications, robust and simple spectrum sensing techniques for OFDM signals receive significant research interest. In this paper, a time domain pilot correlation (TDPC) technique, which overcomes the complexity of FFT-based pilot sensing methods, is proposed. The TDPC technique provides an efficient and simple spectrum sensing scheme for OFDM signals using frequency domain in-band pilots. The proposed technique is based on the cyclic correlation between the complex conjugate product of adjacent OFDM signal segments and two orthogonal local references deriving from the time domain pilot sequence. Interference mitigation techniques, including periodic segmentation of the received OFDM signal, complex conjugate multiplication-based phase rotation locking, and magnitude-based correlation ratio, are adopted in this algorithm to improve the sensing performance. It is validated by simulations that the TDPC scheme achieves satisfactory sensing reliability at low SNR and is robust to both timing and frequency offsets.
\end{abstract}

\section{INTRODUCTION}

Spectrum sensing is one fundamental enabling technology for cognitive radio (CR) communications, which is emerging as a promising technology to improve the spectrum utilization efficiency based on the opportunistic spectrum access strategy [1]. Orthogonal frequency division multiplexing (OFDM), which utilizes a number of orthogonal subcarriers to transmit parallel data streams, is adopted by various communication applications [2], [3]. It is also one of primary transmission technologies for CR communications due to its high spectral efficiency, variable transmission parameters and robustness to multipath channel distortion. As a result, reliable spectrum sensing techniques for OFDM signals with low complexity are of significant importance.

Spectrum sensing for OFDM signal has received substantial attention from both academia and industry. Spectrum sensing techniques for OFDM can be generally classified into two categories: blind methods and non-blind methods [4]. Energy detection is a typical blind method which makes a decision based on the estimation of the energy of the received signals [5]. Non-blind methods are based on the features of the OFDM signals. Typical features exploited for the spectrum sensing are the cyclic prefix (CP) and the in-band pilot used in the OFDM system. The sensing performance of CP-based spectrum sensing methods depends highly on the length of CP [6], [7]. When the duration of CP is short, a long sensing period is required to achieve good sensing performance. Fast Fourier transform
(FFT) based pilot spectrum sensing algorithms with good sensing performance are proposed in [8], [9]. However, their implementation is limited by the computational complexity of the FFT algorithm in the sensing process. In [10], a time domain symbol cross-correlation method for pilot inserted OFDM signal is presented. Nevertheless, the sensing threshold selection in their algorithm is related to the estimation of the noise variance. The sensing performance is directly related to the reliability of the estimated noise parameters.

In this paper, a time domain pilot correlation (TDPC) based spectrum sensing technique is proposed for the frequency domain pilot inserted OFDM. The proposed technique is based on the cyclic correlation between the complex conjugate product of two adjacent received OFDM signal segments and two orthogonal local references, which are derived from the pilot sequence in time domain. Timing offset is approximately compensated by the cyclic correlation due to the periodicity of the time domain pilot sequence when the primary signal is present. The impact of noise to sensing reliability is mitigated by the time domain segment averaging following the complex conjugate multiplication (CCM) based phase rotation locking processing. The robustness to frequency offset is improved by considering only the magnitude of the correlation function, because frequency offset results in a phase rotation to the time domain OFDM signal. In addition, since the correlations are carried out on identical distorted multiplication results, their magnitude ratio, which is the sensing metric adopted in the TDPC algorithm, requires a noise power insensitive threshold.

The organization of this paper is as follows. The OFDM system model considered in this paper is introduced in Section II. The proposed TDPC spectrum sensing algorithm and corresponding interference mitigation techniques are presented in Section III. Simulation results are provided to validate the analysis and evaluate the performance of the proposed sensing algorithm in Section IV. The paper is concluded in Section V.

\section{SYSTEM MODEL}

In an OFDM system, in-band pilots in frequency domain are usually embedded into the OFDM signal for channel estimation and synchronization purposes. The total of $N$ subcarriers of an OFDM system can be divided into two sets: data subcarriers $D(k)$ and pilot subcarriers $P(k)$. The time domain OFDM signal with $N$-point inverse fast Fourier 
transform (IFFT) can be expressed as:

$$
x(n)=d(n)+p(n),
$$

where

$$
\begin{aligned}
& d(n)=\frac{1}{\sqrt{N}} \sum_{k \in \mathcal{D}} D(k) W_{N}^{k n}, \\
& p(n)=\frac{1}{\sqrt{N}} \sum_{k \in \mathcal{P}} P(k) W_{N}^{k n},
\end{aligned}
$$

and

$$
W_{N}=\exp (j 2 \pi / N) .
$$

$\mathcal{D}$ denotes the set of data subcarriers and $\mathcal{P}$ denotes the set of pilot subcarriers. Therefore, the time domain OFDM signal can be taken as the sum of two components: time domain data-carrying sequence and time domain pilot sequence.

With the consideration of the timing offset and frequency offset between the OFDM transmitter and the sensing device, the received signal at the sensing device can be written as:

$$
\begin{array}{r}
r(n)=d\left(n-\tau_{0}\right) W_{N}^{\left(n-\tau_{0}\right) \varepsilon}+p\left(n-\tau_{0}\right) W_{N}^{\left(n-\tau_{0}\right) \varepsilon}+w(n), \\
n=0,1, \cdots, N_{s}-1 .
\end{array}
$$

$\tau_{0}$ represents the timing offset in terms of signal samples; $\varepsilon$ is the frequency offset normalized by the subcarrier spacing; $w(n)$ denotes the additive white Gaussian noise (AWGN) with a mean of 0 and a variance of $\sigma_{w}^{2} . N_{s}$ is the total symbol duration of each transmitted OFDM symbol while the length of the CP is $N_{C P}$, i.e. $N_{s}=N+N_{C P}$.

\section{Proposed TDPC Sensing TeChNiQue}

\section{A. Properties of the Time Domain Data and Pilot Sequences}

Since the data sequence $D(k)$ in (2) is randomly generated, when the size of $\mathcal{D}$ is large, the central limit theorem (CLT) can be invoked and the time domain data signal $d(n)$ can be considered as a zero-mean Gaussian distributed variable with a variance of $\sigma_{d}^{2}$. Due to the properties of Gaussian distribution, the received data-carrying signal with both timing offset and frequency offset $d\left(n-\tau_{0}\right) W_{N}^{\left(n-\tau_{0}\right) \varepsilon}$ also follows a zero-mean Gaussian distribution with variance $\sigma_{d}^{2}$. Assume that invariant pilots are inserted at fixed subcarriers in the frequency domain for all the transmitted OFDM signals, the time domain pilot sequence provides a distinctive pattern for the spectrum sensing of OFDM signals. Based on the properties of time domain data and pilot signals, the received signal $r(n)$ can be represented as:

$$
r(n)=p\left(n-\tau_{0}\right) W_{N}^{\left(n-\tau_{0}\right) \varepsilon}+\hat{w}(n) .
$$

Here the time domain data-carrying signal is treated as Gaussian noise to the corresponding pilot sequence. $\hat{w}(n)$ denotes the combined interference, which is Gaussian distributed with a mean of 0 and a variance of $\sigma_{w}^{2}+\sigma_{d}^{2}$.

\section{B. Interference Mitigation Techniques}

Due to severe attenuation caused by signal fading and shadowing, the SNR of OFDM signals received at the sensing device may be very low. In addition, since the existence of the primary signal is unknown prior to the sensing process, timing and frequency synchronization is unavailable before the sensing operation. Therefore, special interference mitigation techniques are implemented to improve the sensing performance in this proposed TDPC algorithm. For unknown timing offset, since the time domain pilot sequence is a periodical signal with a period of $N_{s}$ and each transmitted OFDM symbol has an identical time domain pilot sequence, each segment with a length of $N_{s}$ still includes a complete time domain pilot sequence but in a circularly rotated order. Time domain averaging can be adopted to mitigate the noise effect at low SNR. However, it is worth to note that since the frequency offset results in a time domain phase rotation to the OFDM signal, direct time domain segment averaging may eliminate the pilot component when segments have inverse phases. Considering the phase rotation between each two adjacent OFDM segments is a constant, complex conjugate multiplication of every two successive OFDM signal segments is implemented before the average operation to lock the damage of the frequency offset.

The first step of this algorithm is to segment the received signals with an equal length of $N_{s}$. For any two successive segments $m$ and $m+1$, we have:

$$
\begin{array}{r}
r_{m}(n)=p\left(n-\tau_{0}\right) W_{N}^{\left(n+(m-1) N_{s}-\tau_{0}\right) \varepsilon}+\hat{w}_{m}(n), \\
r_{m+1}(n)=p\left(n-\tau_{0}\right) W_{N}^{\left(n+m N_{s}-\tau_{0}\right) \varepsilon}+\hat{w}_{m+1}(n), \\
n=0,1, \cdots, N_{s}-1 .
\end{array}
$$

Then the CCM is employed:

$$
\begin{aligned}
Z_{m}(n)= & r_{m}^{*}(n) \times r_{m+1}(n) \\
= & p^{*}\left(n-\tau_{0}\right) p\left(n-\tau_{0}\right) W_{N}^{N_{s} \varepsilon} \\
& +p^{*}\left(n-\tau_{0}\right) \hat{w}_{m+1}(n) W_{N}^{-\left(n+(m-1) N_{s}-\tau_{0}\right) \varepsilon} \\
& +p\left(n-\tau_{0}\right) \hat{w}_{m}^{*}(n) W_{N}^{\left(n+m N_{s}-\tau_{0}\right) \varepsilon} \\
& +\hat{w}_{m}^{*}(n) \hat{w}_{m+1}(n)
\end{aligned}
$$

Further simplification gives:

$$
Z_{m}(n)=\left|p\left(n-\tau_{0}\right)\right|^{2} W_{N}^{N_{s} \varepsilon}+w_{m}^{\prime}(n),
$$

where $w_{m}^{\prime}(n)$ is the total noise after CCM. The averaging of $Z_{m}(n)$ calculated over $G$ sets of successive OFDM segment pairs is:

$$
\begin{aligned}
\bar{Z}(n) & =\frac{1}{G} \sum_{m=0}^{G-1} Z_{2 m}(n) \\
& =\left|p\left(n-\tau_{0}\right)\right|^{2} W_{N}^{N_{s} \varepsilon}+\bar{w}_{m}^{\prime}(n) .
\end{aligned}
$$

Note that the total number of segments employed in this calculation is $M=2 G$. It can be seen from (11) that the mean of $\bar{Z}(n)$ is unchanged no matter how many multiplication results are accumulated while the variance of the noise term $\bar{w}_{m}^{\prime}(n)$ is inversely proportional to $G$. As long as the accumulated 
number $G$ is large, the effect of the noise term $\bar{w}_{m}^{\prime}(n)$ in $\bar{Z}(n)$ will be significantly mitigated. As a result, we are able to achieve a reliable spectrum sensing at low SNR environment.

\section{Proposed Sensing Metric}

In the proposed sensing algorithm, a pair of orthogonal local references, $L_{1}(n)$ and $L_{2}(n)$, is generated based on the time domain pilot sequence which is available at the sensing device. The reason to employ two local references is to reduce the sensitivity of sensing threshold to the noise knowledge by employing the magnitude ratio of correlations between these two orthogonal local references and one identical distorted multiplication $\bar{Z}(n)$.

The first local reference $L_{1}(n)$ is the power of the time domain pilot sequence with a complete duration of $N_{s}$ :

$$
L_{1}(n)=|p(n)|^{2}, \quad n=0,1, \cdots, N_{s}-1 .
$$

The other local reference $L_{2}(n)$ is orthogonal to $L_{1}(n)$ and has the same statical nature as $L_{1}(n)$. Therefore,

$$
\sum_{n=0}^{N_{s}-1} L_{2}(n) \times L_{1}(n)=\sum_{n=0}^{N_{s}-1} L_{2}(n) \times|p(n)|^{2}=0 .
$$

Since the generation of orthogonal sequence is not the focus of this paper, we will not go into details for the derivation of the orthogonal local reference $L_{2}(n)$. Ideally, when these two local references are correlated with the averaged complex conjugate product $\bar{Z}(n)$ without synchronization error, there is a significant correlation difference.

In practice, there is potential timing offset between the transmitted and received time domain pilot sequences. Considering that the averaged complex conjugate product $\bar{Z}(n)$ in (11) includes the power of a complete time domain pilot sequence in a circularly rotated order due to the effect of timing offset, the timing offset can be estimated using the cyclic correlation between $\bar{Z}(n)$ and the local reference $L_{1}(n)$ when the primary OFDM signal presents. The cyclic correlation between $\bar{Z}(n)$ and $L_{1}(n)$ can be calculated as:

$$
\begin{gathered}
T_{1}(\nu)=\frac{1}{N_{s}} \sum_{n=0}^{N_{s}-1} L_{1}(n)\left|p\left(\bmod \left\{\frac{n-\tau_{0}+\nu}{N_{s}}\right\}\right)\right|^{2} W_{N}^{N_{s} \varepsilon} \\
+\frac{1}{N_{s}} \sum_{n=0}^{N_{s}-1} L_{1}(n) \bar{w}_{m}^{\prime}\left(\bmod \left\{\frac{n+\nu}{N_{s}}\right\}\right) \\
\nu=0,1, \cdots, N_{s}-1 .
\end{gathered}
$$

$\bmod \{\cdot\}$ denotes the modulo operation. When $\nu=\tau_{0}$, a correlation peak can be observed, that is:

$$
\begin{aligned}
T_{1}\left(\tau_{0}\right)= & \frac{1}{N_{s}} \sum_{n=0}^{N_{s}-1}|p(n)|^{4} W_{N}^{N_{s} \varepsilon} \\
& +\frac{1}{N_{s}} \sum_{n=0}^{N_{s}-1}|p(n)|^{2} \bar{w}_{m}^{\prime}\left(n+\tau_{0}\right) .
\end{aligned}
$$

In order to further mitigate the effect of the frequency offset, which results a phase rotation in the time domain OFDM signal, the magnitude of $T_{1}\left(\tau_{0}\right)$ is adopted in this algorithm, that is:

$$
\left|T_{1}\left(\tau_{0}\right)\right|=\sqrt{\left(\frac{1}{N_{s}} \sum_{n=0}^{N_{s}-1}|p(n)|^{4}\right)^{2}+V}
$$

where $V$ denotes the combining noise:

$$
\begin{aligned}
V= & \frac{1}{N_{s}^{2}} \sum_{n=0}^{N_{s}-1}|p(n)|^{4} W_{N}^{N_{s} \varepsilon} \sum_{n=0}^{N_{s}-1}|p(n)|^{2} \bar{w}_{m}^{\prime *}\left(n+\tau_{0}\right) \\
& +\frac{1}{N_{s}^{2}} \sum_{n=0}^{N_{s}-1}|p(n)|^{4} W_{N}^{-N_{s} \varepsilon} \sum_{n=0}^{N_{s}-1}|p(n)|^{2} \bar{w}_{m}^{\prime}\left(n+\tau_{0}\right) \\
& +\frac{1}{N_{s}^{2}} \sum_{n=0}^{N_{s}-1}|p(n)|^{2} \bar{w}_{m}^{\prime *}\left(n+\tau_{0}\right) \sum_{n=0}^{N_{s}-1}|p(n)|^{2} \bar{w}_{m}^{\prime}\left(n+\tau_{0}\right) .
\end{aligned}
$$

A significant correlation peak can still be observed though it is influenced by the noise. As mentioned before, since the variance of the noise term $\bar{w}_{m}^{\prime}(n)$ is significantly reduced according to the averaging process, the effect of this combining noise $V$ as well as the phase rotation $W_{N}^{N_{s} \varepsilon}$ which only acts on the noise term in (16), is also remarkably mitigated. The robustness to frequency offset is further improved.

The timing offset $\tau_{0}$ can therefore be estimated by selecting the maximum correlation from $\left|T_{1}(\nu)\right|$, mathematically,

$$
\left|T_{1}(\nu)\right|_{\nu=\tau}=\max \left\{\left|T_{1}(\nu)\right|\right\},
$$

where $\tau$ denotes the estimation of timing offset $\tau_{0}$. Following this, the local reference $L_{2}(n)$ is correlated with the synchronized multiplication result $\bar{Z}(n+\tau)$, the magnitude of this correlation becomes:

$$
\left|T_{2}(\tau)\right|=\left|\frac{1}{N_{s}} \sum_{n=0}^{N_{s}-1} L_{2}(n) \bar{w}_{m}^{\prime}(n+\tau)\right| .
$$

The sensing metric $\gamma$, which is the ratio between the magnitude of $T_{1}(\tau)$ and the magnitude of $T_{2}(\tau)$, is obtained as:

$$
\gamma=\frac{\left|T_{1}(\tau)\right|}{\left|T_{2}(\tau)\right|} .
$$

On the other hand, when primary OFDM signals are absent, only noise exists. Received segments $m$ and $m+1$ will be:

$$
\begin{aligned}
& r_{m}^{\prime}(n)=w_{m}(n), \quad n=0,1, \cdots, N_{s}-1, \\
& r_{m+1}^{\prime}(n)=w_{m+1}(n), \quad n=0,1, \cdots, N_{s}-1 .
\end{aligned}
$$

After CCM, the averaged multiplication result over $G$ sets of successive segment pairs can be given by:

$$
\bar{Z}^{\prime}(n)=\frac{1}{G} \sum_{m=0}^{G-1} w_{2 m}^{*}(n) w_{2 m+1}(n) .
$$

Similarly, the sensing metric when OFDM signal is not active, with the magnitude correlations $T_{1}^{\prime}\left(\tau^{\prime}\right)$ and $T_{2}^{\prime}\left(\tau^{\prime}\right)$, can be formulated as:

$$
\gamma^{\prime}=\frac{\left|T_{1}^{\prime}\left(\tau^{\prime}\right)\right|}{\left|T_{2}^{\prime}\left(\tau^{\prime}\right)\right|}
$$


where

$$
\left|T_{1}^{\prime}(\nu)\right|_{\nu=\tau^{\prime}}=\max \left\{\left|T_{1}^{\prime}(\nu)\right|\right\}
$$

Comparing $\gamma$ with $\gamma^{\prime}$, we can find that $\left|T_{1}(\tau)\right|$ and $\left|T_{1}^{\prime}\left(\tau^{\prime}\right)\right|$ will always provide distinct values due to the different correlation natures, i.e. the correlation between local reference and the related OFDM signal, and the correlation between the local reference and independent noise. Meanwhile, $\left|T_{2}(\tau)\right|$ and $\left|T_{2}^{\prime}\left(\tau^{\prime}\right)\right|$ have close values due to their similar statistical characteristics. Thus, a relative high ratio can be observed when the OFDM signal is present while a small ratio is achieved when the OFDM signal is absent. The proposed sensing metric $\gamma$ can be used to identify the existence of the primary OFDM signals.

\section{Sensing Threshold Selection}

The signal presence is determined by comparing the sensing metric against a certain threshold $\lambda$. If $\gamma>\lambda$, primary users are deemed to be present. Otherwise, the spectrum channel of interest is determined as vacant. In the proposed sensing algorithm, the sensing threshold is selected with respect to the false alarm probability $P_{f a}$, i.e. the probability that a false decision is made when primary OFDM signals are absent.

For the segment CCM result $\bar{Z}^{\prime}(n)$ in (23), it is Gaussian distributed with a mean of 0 and a variance of $\frac{1}{G} \sigma_{w}^{4}$ according to the central limit theorem. For the term $T_{2}^{\prime}\left(\tau^{\prime}\right)$, due to the property of Gaussian distribution, it is Gaussian distributed with a mean of 0 and a variance of $\frac{\sum_{n=0}^{N_{s}-1} L_{2}^{2}(n)}{G N_{s}^{2}} \sigma_{w}^{4}$. As the magnitude of complex Gaussian distributed variable following Rayleigh distribution, it can be asserted:

$$
\left|T_{2}^{\prime}\left(\tau^{\prime}\right)\right| \sim \text { Rayleigh }\left(\sqrt{\frac{\sum_{n=0}^{N_{s}-1} L_{2}^{2}(n)}{2 G N_{s}^{2}}} \sigma_{w}^{2}\right) .
$$

Since the value of $N_{s}$ is usually large, $2 G N_{s}^{2}$ will be a large value especially when we observe a large number of segments in the sensing process. The variance of this Rayleigh distributed variable will be very small. As a result, $\left|T_{2}^{\prime}\left(\tau^{\prime}\right)\right|$ can be approximated by the mean of this Rayleigh distribution, it yields:

$$
\gamma^{\prime}=\max \left|\frac{T_{1}^{\prime}(\nu)}{\sqrt{\frac{\pi \sum_{n=0}^{N_{s}-1} L_{2}^{2}(n)}{4 G N_{s}^{2}}} \sigma_{w}^{2}}\right| .
$$

With similar analysis, $T_{1}^{\prime}(\nu)$ is also Gaussian distributed with a mean of 0 and a variance of $\frac{\sum_{n=0}^{N_{s}-1} L_{1}^{2}(n)}{G N_{s}^{2}} \sigma_{w}^{4}$. As a result,

$$
\left|\frac{T_{1}^{\prime}(\nu)}{\sqrt{\frac{\pi \sum_{n=0}^{N_{s}-1} L_{2}^{2}(n)}{4 G N_{s}^{2}}} \sigma_{w}^{2}}\right| \sim \text { Rayleigh }\left(\sqrt{\frac{2 \sum_{n=0}^{N_{s}-1} L_{1}^{2}(n)}{\pi \sum_{n=0}^{N_{s}-1} L_{2}^{2}(n)}}\right) .
$$

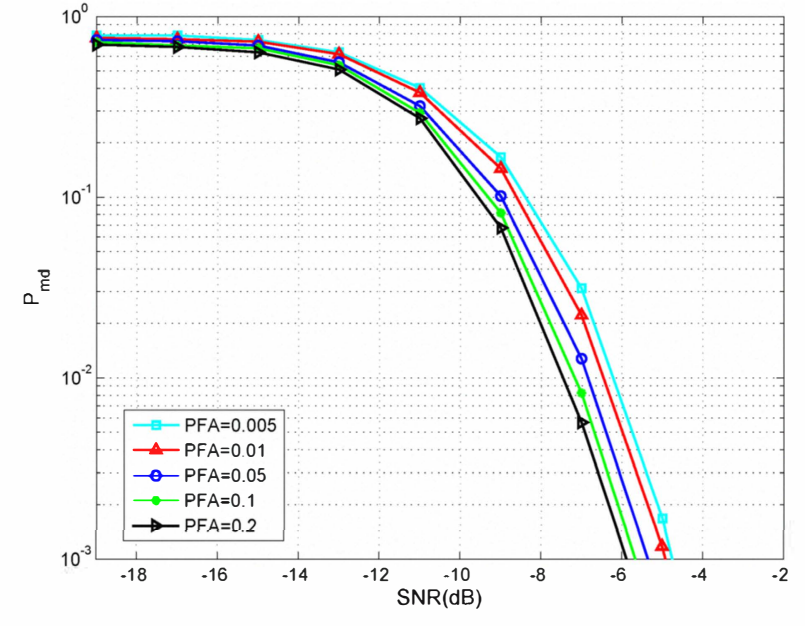

Fig. 1. Probability of miss detection under different false alarm requirements with $M=100, \mathrm{CFO}=0.1$ and random timing offset.

The false alarm probability $P_{f a}$ with respect to $\gamma^{\prime}$ can be represented as:

$$
\begin{aligned}
P_{f a} & =\operatorname{Prob}\left(\gamma^{\prime}>\lambda\right) \\
& =1-F_{\text {ray }}\left(\lambda ; \sqrt{\frac{2 \sum_{n=0}^{N_{s}-1} L_{1}^{2}(n)}{\pi \sum_{n=0}^{N_{s}-1} L_{2}^{2}(n)}}\right)^{N_{s}},
\end{aligned}
$$

where $F_{r a y}(x ; \sigma)$ denotes the Rayleigh cumulative distribution function,

$$
F_{\text {ray }}(x ; \sigma)=\int_{-\infty}^{x} \frac{u}{\sigma^{2}} \exp \left(\frac{-u^{2}}{2 \sigma^{2}}\right) d u .
$$

Based on a required false alarm probability $P_{f a}$, the threshold $\lambda$ can be selected, that is:

$$
\lambda=\operatorname{raylinv}\left(\left(1-P_{f a}\right)^{1 / N_{s}}, \sqrt{\frac{2 \sum_{n=0}^{N_{s}-1} L_{1}^{2}(n)}{\pi \sum_{n=0}^{N_{s}-1} L_{2}^{2}(n)}}\right),
$$

where $\operatorname{raylinv}(y, \sigma)$ is the inverse of the Rayleigh cumulative distribution function. We can find from (31) that no noise variance knowledge is required in the threshold selection for the proposed sensing metric.

\section{SimULATION RESULTS}

Simulations are carried out to evaluate the performance of the proposed OFDM signal time domain pilot correlation sensing algorithm. The robustness of the proposed TDPC sensing algorithm to the noise, timing offset and frequency offset are simulated using Matlab. An OFDM system with 256 subcarriers is employed in these simulations. Without further specification, 16 pilots are inserted into each OFDM signal in the frequency domain; the false alarm probability requirement is set to 0.1 ; the frequency offset is assumed to be 0.1 and the timing offset is randomly generated by Matlab. 


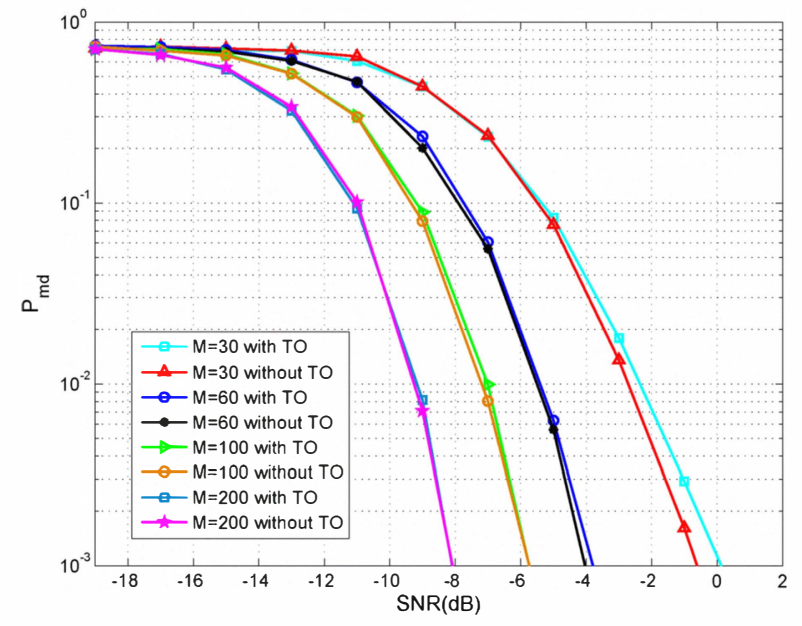

Fig. 2. Robustness of the proposed algorithm to timing offset while $P_{f a}=$ 0.1 and $\mathrm{CFO}=0.1$.

Fig. 1 evaluates the miss detection probability $P_{m d}$ of the proposed TDPC algorithm with different false alarm probability requirements. 100 segments of the received signals are adopted in the complex conjugate multiplication, i.e. 50 multiplication results are averaged in the sensing processing. As shown in the figure, with a lower requirement of $P_{f a}$, i.e. larger false alarm probability, a better miss detection probability can be observed. Meanwhile, a miss detection probability less than 0.001 can always be achieved when SNR is beyond $-5.2 d B$ in this simulation environment.

The robustness of the proposed algorithm to timing and frequency offsets is simulated and shown in Fig. 2 and Fig. 3 , respectively. In Fig. 2, we evaluate the $P_{m d}$ of the sensing algorithm with and without random timing offset between the transmitter and the sensing device observing different numbers of received segments. It can be concluded from the simulation results that the sensing performance only experiences little mitigation when there is random timing offset, especially when more segments are observed in the sensing process to further mitigate the effect of the noise. Meanwhile, when more segments are observed in the sensing process, the noise effect on the sensing reliability can be further mitigated. In order to study the robustness to the frequency offset, carrier frequency offsets (CFO) between the transmitted and received signals are set to $0.01,0.1$ and 2 in the simulations. Undistinguishable sensing performances in terms of miss detection probability are observed in Fig. 3.

\section{CONCLUSION}

In order to overcome the inefficiency of FFT-based pilot spectrum sensing methods, a time domain pilot correlation sensing technique is proposed for OFDM signal in this paper. The correlations between the CCM of adjacent OFDM symbols and two pilot-based orthogonal local references in time domain are used to detect the presence of primary OFDM signals. The noise effect to the sensing reliability is

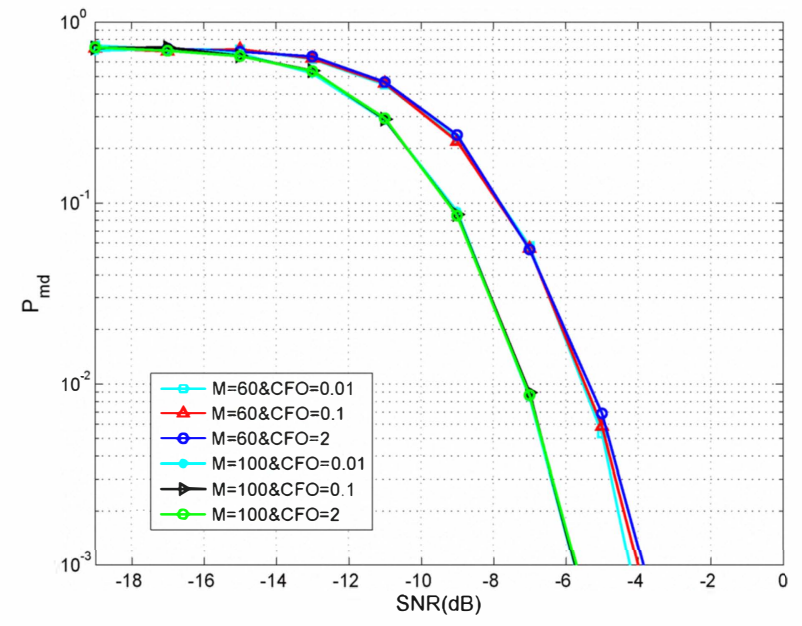

Fig. 3. Robustness of the proposed algorithm to frequency offset with $P_{f a}=$ 0.1 and random timing offset.

mitigated by a time domain segment averaging following the phase rotation locking processing. The robustness to timing offset is improved by the time domain OFDM symbol length based segmentation and the cyclic correlation. The robustness to frequency offset is improved by the CCM of adjacent OFDM symbols and the use of the correlation magnitude. In addition, the sensitivity of the sensing metric threshold to noise is reduced by using the correlation magnitude ratio. Simulation results show that the sensing performance of the proposed technique is satisfactory under low SNR condition with presence of both timing and frequency offsets.

\section{REFERENCES}

[1] T. Yucek and H. Arslan, "A Survey of Spectrum Sensing Algorithms for Cognitive Radio Applications," IEEE Communications Surveys \& Tutorials, vol. 11, no. 1, pp. 116-130, 2009.

[2] ETSI, "Digital Video Broadcasting: Framing Structure, Channel Coding, and Modulation for Digital Terrestrial Television," European Telecommunication Standard EN300744, Aug. 1997.

[3] IEEE Standard, "The Standard for Local and Metropolitan Area Networks C Part 16: Air Interface for Fixed Broadband Wireless Access Systems," Jun. 2004.

[4] N. Kundargi and A. Tewfik, "Sequential Pilot Sensing of ATSC Signals in IEEE 802.22 Cognitive Radio Networks," in Proc. IEEE Intern. Conf. Acoustics, Speech and Signal Processing, 2008, pp. 2789-2792.

[5] J. E. Slat and H. H. Nquyen, "Performance Prediction for Energy Detection of Unknown Signals," IEEE Trans. Vehicular Technology, vol. 57 , no. 6 , pp. 3900-3904, Nov. 2008.

[6] T. Yucek and H. Arslan, "OFDM Signal Identification and Transmission Parameter Estimation for Cognitive Radio Applications," in Proc. IEEE Global Telecommunication Conf., Dec. 2007, pp. 4056-4060.

[7] S. Chaudhari, V. Koivunen and H. V. Poor, "Autocorrelation-Based Decentralized Sequential Detection of OFDM Signals in Cognitive Radios," IEEE Trans. Signal Processing, vol. 57, no. 7, pp. 2690-2700, 2009.

[8] H. Li, X. Wang, C. Wang and J.-Y. Chouinard, "Robust Spectrum Sensing of OFDM Signal without Noise Variance Knowledge," in Proc. IEEE Canadian Workshop on Information Theory, 2009, pp. 91-94.

[9] H.-W. Chen, X. Wang, C.-L. Wang and H. Lin, "Spectrum Sensing of Unsynchronized OFDM Signals for Cognitive Radio Communications," in Proc. IEEE Vehicular Technology Conf., 2009, pp. 1-5.

[10] H.-S. Chen, W. Gao and D. G. Daut, "Spectrum Sesning for OFDM Systems Employing Pilot Tones," IEEE Trans. Wireless Communications, vol. 8, no. 12, pp. 5862-5870, Dec. 2009. 